\title{
Carotenoid Analysis of Cassava Genotypes Roots (Manihot Esculenta Crantz) Cultivated in Southern Brazil Using Chemometric Tools
}

\author{
Rodolfo Moresco, Virgílio G. Uarrota, Aline Pereira, \\ Maíra Tomazzoli, Eduardo da C. Nunes, \\ Luiz Augusto Martins Peruch, Christopher Costa, Miguel Rocha \\ and Marcelo Maraschin
}

\begin{abstract}
Manihot esculenta roots rich in $\beta$-carotene are an important staple food for populations with risk of vitamin A deficiency. Cassava genotypes with high provitamin A activity have been identified as a strategy to reduce the prevalence of deficiency of this vitamin, In this study, the metabolomics characterization focusing on the carotenoid composition of ten cassava genotypes cultivated in southern Brazil by UV-visible scanning spectrophotometry and reverse phase-high performance liquid chromatography was performed. The data set was used for the construction of a descriptive model by chemometric analysis. The genotypes of yellow roots were clustered by the higher concentrations of cis- $\beta$-carotene and lutein. Inversely, cream roots genotypes were grouped precisely due to their lower concentrations of these pigments, as samples rich in lycopene differed among the studied genotypes. The analytical approach (UV-Vis, HPLC, and chemometrics) used showed to be efficient for understanding the chemodiversity of cassava genotypes, allowing to classify them according to important features for human health and nutrition.
\end{abstract}

Keywords Chemometrics - Descriptive models • Partial metabolome • Cassava genotypes $\cdot$ Carotenoids $\cdot$ RP-HPLC $\cdot$ UV-vis

\footnotetext{
R. Moresco ( $)$ - V.G. Uarrota · A. Pereira · M. Tomazzoli · M. Maraschin

Plant Morphogenesis and Biochemistry Laboratory, Federal University of Santa Catarina,

Florianopolis, Brazil

e-mail: rodolfo.moresco@posgrad.ufsc.br

E. da C. Nunes · L.A.M. Peruch

Santa Catarina State Agricultural Research and Rural Extension Agency (EPAGRI),

Experimental Station of Urussanga, Urussanga, Brazil

C. Costa $\cdot$ M. Rocha

Centre Biological Engineering School of Engineering, University of Minho, Braga, Portugal

(C) Springer International Publishing Switzerland 2015

R. Overbeek et al. (eds.), 9th International Conference on Practical Applications

of Computational Biology and Bioinformatics, Advances in Intelligent Systems

and Computing 375, DOI 10.1007/978-3-319-19776-0_2
} 


\section{Introduction}

Cassava (Manihot esculenta Crantz, 1766) currently ranks as the third most important species as a source of calories in the world among the group of staple food crops, including rice and maize. It is primarily consumed in places where there are prevailing drought, poverty, and malnutrition [1]. Diseases related to vitamin A deficiency are among the major nutritional problems in developing countries. It is estimated that 190 million children in preschool age have low retinol activity in plasma $\left(<0.70 \mu \mathrm{mol} . \mathrm{L}^{-1}\right)$, subclinical symptom of this deficiency [2]. Cassava is considered a staple food for many populations with risk of vitamin A deficiency and is predominantly produced by small-scale farmers with limited resources. In cassava, $\beta$-carotene is the majoritarian pro-vitamin A carotenoid [3], but the amounts found in white and cream cassava roots (the most commonly consumed by populations) are low [4]. It is known that roots with yellow flesh are highly correlated with the concentration of carotenoids and the search for materials with higher provitamin A activity is recognized as a strategy to reduce the prevalence of this deficiency [5]. Besides $\beta$-carotene, $M$. esculenta also contains small amounts of other carotenoids, e.g., lycopene and the xanthophylls, lutein and $\beta$-cryptoxanthin, with recognized health benefits [6].

Because of the high importance of cassava crops in Brazil, genebanks of cassava collections have been established and maintained for the purpose of preserving commercial varieties, traditional landraces and wild genotypes. Thus, the identification and preservation of genotypes with high carotenoid concentrations is thought to be relevant for the Brazilian and global food security and nutrition.

This study, in connection with the metabolomics characterization of the genebank's cassava accesses, emphasizes their carotenoid profile in root samples, using a typical analytical platform, i.e., UV-visible spectrophotometry (UV-Vis) and reverse phasehigh performance liquid chromatography (RP-HPLC). The data set afforded (i.e., the spectrophotometric profiles and the chromatographic quantification of each carotenoid compound) was used to build descriptive and classification models by calculation of the principal components and cluster analysis. Such an analytical approach allows the rapid and effective extraction of relevant and non-redundant information from a set of complex data, enabling a more detailed and robust understanding of possible differences and/or similarities in the studied samples, as well as their improved discrimination. In practical terms, this study develops and applies biotechnological approaches, by coupling the use of biochemical markers with bioinformatics tools in order to gain insights to support genetic breeding programs of cassava.

\section{Materials and Methods}

Roots of ten genotypes of $M$. esculenta (2010/2011 season) from the EPAGRI's germplasm bank (Urussanga Experimental Station, 28³1'18' 'S, 49¹9'03' 'W, Santa Catarina, southern Brazil) were used in this study. Traditionally, they have been called 
Apronta mesa, Pioneira, Oriental, Amarela, Catarina, IAC, Salézio, Estação, Videira and Rosada and were selected based on their economic and social importance. Carotenoids were extracted from fresh roots as described by Rodriguez-Amaya \& Kimura (2004) [7] using an Ultra-Turrax (Janke \& Kunkel IKA - T25 basic) and organic solvents: acetone and petroleum ether. The absorbances of the organosolvent extracts were collected on a UV-visible spectrophotometer (Gold Spectrum lab $53 \mathrm{UV}-\mathrm{V}$ is spectrophotometer, BEL photonics, Brazil) using a spectral window from 200 to $700 \mathrm{~nm}$. Aliquots $(10 \mu \mathrm{l})$ of the extracts were also injected into a liquid chromatograph (LC-10A Shimadzu) system equipped with a C18 reversed-phase column (Vydac 201TP54, $250 \mathrm{~mm} \times 4.6 \mathrm{~mm}, 5 \mu \mathrm{m} \varnothing, 35^{\circ} \mathrm{C}$ ) coupled to a precolumn (C18 Vydac 201TP54, $30 \mathrm{~mm} \times 4.6 \mathrm{~mm}, 5 \mu \mathrm{m} \varnothing)$ and a spectrophotometric detector $(450 \mathrm{~nm})$. Methanol: acetonitrile (90: 10, v/v) was used for elution at a rate of $1 \mathrm{ml} / \mathrm{min}$. The identification and quantification of compounds of interest was carried out via co-chromatography and comparison of retention times of samples with those of standard compounds (Sigma-Aldrich, USA) under the same experimental conditions.

Data were collected, summarized, and submitted to analysis of variance (ANOVA) followed by post hoc Tukey's test $(p<0.05)$ for mean comparison. All procedures were performed in triplicate $(n=3)$. The processing of the spectrophotometric profile considered the definition of the spectral window of interest (200-700 nm), baseline correction, normalization, and optimization of the signal/noise ratio (smoothing). The processed data set was initially subjected to multivariate statistical analysis, by applying principal component analysis (PCA) and clustering methods. Furthermore, the spectral data set and the amounts of the target carotenoids determined by RP-HPLC were used for calculation of the principal components, supported by scripts written in R language (v. 3.1.1) [8] using tools defined by our research group and some functions from the packages Chemospec [9], HyperSpec [10], and ggplot2. All R scripts, raw data, and additional chemometric analysis are available in supplementary material, in http://darwin.di. uminho.pt/metabolomicspackage/ as well as the data analysis report automatically generated from the $\mathrm{R}$ scripts using the features provided by $\mathrm{R}$ Markdown (http:// darwin.di.uminho.pt/metabolomicspackage/cassava-carotenoids.html). This allows anyone to fully reproduce and document the experiments.

\section{Results and Discussion}

Carotenoids show typically maximum absorption at $450 \mathrm{~nm}$ [7] and as depicted in Fig. 1, all the spectral profiles $(200-700 \mathrm{~nm})$ of the yellow and red cassava root extracts revealed prominent absorbance peaks between $400-500 \mathrm{~nm}$, indicating that the organosolvent system used was efficient to extract the target metabolites. Lower absorbance values were found in cream color-roots, precisely because they have low concentrations of carotenoids as the Rosada genotype (pink root) showed the highest absorbance values at $450 \mathrm{~nm}$. 


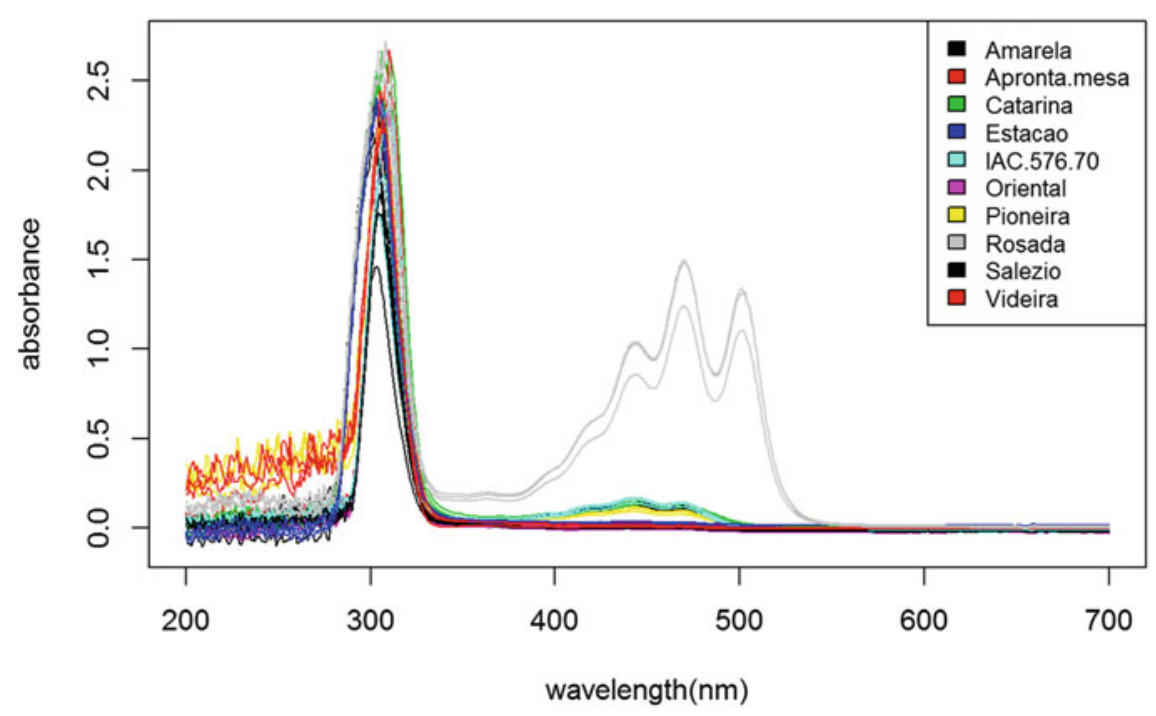

Fig. 1 Typical UV-Vis spectrophotometric profiles $(\lambda=200-700 \mathrm{~nm}$, acetone: petroleum ether $\mathrm{v} / \mathrm{v}$ ) of root parenchymal tissues of ten cassava genotypes cultivated in southern Brazil

When the principal components were calculated from the full spectrophotometric profile $(\lambda=200-700 \mathrm{~nm})$ data matrix, PC1 and PC2 contributed to explain $78.9 \%$ of the total variance of the data set. However, a clear discrimination of the samples according to the carotenoid concentrations was not found. Only the Rosada genotype distinguished from the others by grouping in PC1 + / PC2 -. Genotypes with high (yellow roots) and low carotenoid contents (cream roots) were spread out over the factorial distribution plane, making difficult to discriminate between them (Fig. 2).

Such findings prompted us to build a second analytical model by applying PCA to the carotenoid fingerprint region of the UV-visible $(400-500 \mathrm{~nm})$. In this case, PC1 and PC2 accounted for $99.97 \%$ of the variance, clearly revealing three groups according to their similarities (Fig. 3). Interestingly, the samples were grouped according to their carotenoids contents determined by RP-HPLC and distributed according to the root flesh color. Cassava genotypes with yellow roots (Pioneira, Amarela, Catarina and IAC576-70) were clustered along the PC2 + axis. Genotypes with cream roots and lower carotenoid content (Apronta mesa, Oriental, Salézio, Estação and Videira) were grouped in PC1/PC2 -. In its turn, Rosada genotype (flesh red) seems to have a metabolic profile occurring away from all the other samples.

The chromatographic profiles of the organosolvent extracts identified cis- and trans$\beta$-carotene, $\alpha$-carotene, lutein, and $\beta$-cryptoxanthin in all the cassava genotypes analyzed. The presence of lycopene, a common precursor of the carotenoids above mentioned, was detected only in Rosada genotype, a fact that led us to speculate this is an important reason for its clear discrimination in respect to other genotypes. 

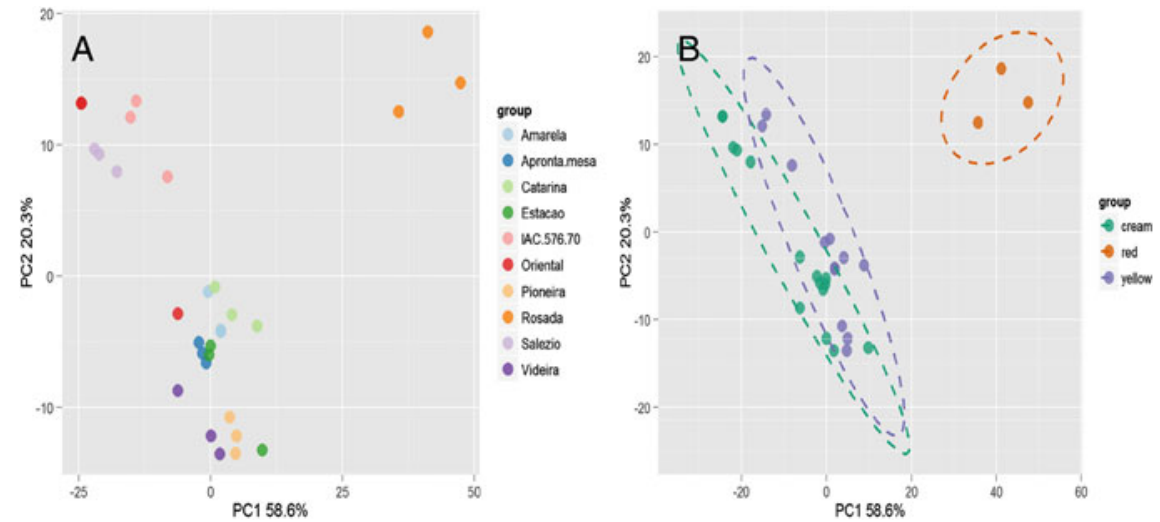

Fig. 2 A - Factorial distribution (principal components 1 and 2) of the spectral data set (UV-Vis, 200-700 nm) of the organosolvent extract of roots of ten cassava genotypes. B - Graphical demonstration according to the root flesh color
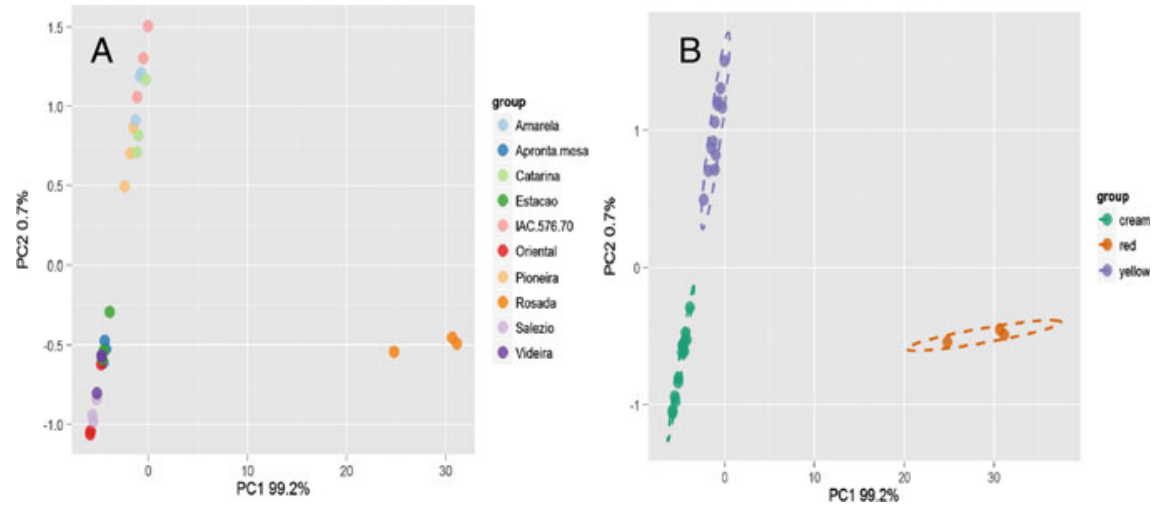

Fig. 3 A - Factorial distribution (principal components 1 and 2) of the spectral data set of the fingerprint region of carotenoids (UV-Vis $400-500 \mathrm{~nm}$, acetone: petroleum ether - v/v). B Graphical demonstration according to the root flesh color

The isomer trans of $\beta$-carotene was the major compound regardless the sample analyzed.

In a second series of experiments, PCA was applied to the chromatographic data set revealing patterns of similarity of carotenoid composition among the studied genotypes. These findings corroborate the results previously found by UV-Vis scanning spectrophotometry taking into account the fingerprint region of carotenoids (i.e. 400-500 nm). Figure 4 depicts the grouping of genotypes after calculation of the principal components from the RP-HPLC quantification of carotenoids. PC1 and PC2 explain $97.8 \%$ of the total variance of the sample population under study. 


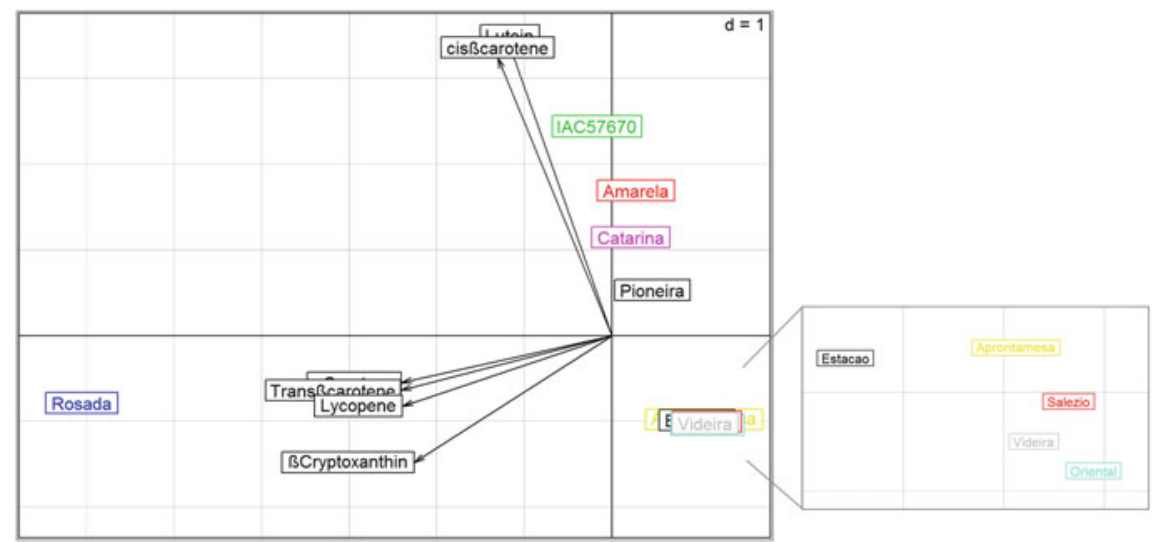

Fig. 4 A - Score-scatter plot (PC1 and PC2) of the quantitative data of carotenoids determined by RP-HPLC in root samples of ten cassava genotypes $(n=3)$. B - Magnification to the overlapping samples at the PCA

The genotypes with yellow roots (Pioneira, Amarela, Catarina, and IAC-576-70) were grouped in $\mathrm{PC} 2+$, influenced by the higher concentration of cis- $\beta$-carotene and lutein. Inversely, the genotypes with cream roots (Apronta mesa, Oriental, Salézio, Estação, and Videira) were grouped in PC1 +/PC2 - due to their lower amounts of these pigments. Samples of red roots (i.e., Rosada) showed higher dissimilarity among the studied genotypes, grouping into PC1/PC2 -. This result seems to be directly influenced by the presence of lycopene and the higher concentrations of trans- $\beta$-carotene, $\alpha$-carotene, and $\beta$-cryptoxanthin. Finally, hierarchical cluster analysis was applied to the chromatographic data, affording similar results to UV-Vis scanning spectrophotometry for the fingerprint region of carotenoids. Genotypes with the highest similarity in their carotenoid composition are represented by cluster hierarchical analysis in Fig. 5. The cophenetic correlation was $97.61 \%$. The similarities were defined based on the Euclidean distance between two samples using the arithmetic average (UPGMA).

\section{Conclusions}

The data set obtained by the analytical techniques employed in this work allowed a better understanding of the chemical variability associated with roots' carotenoid composition of the cassava genotypes. The large disparity in carotenoid contents reveals that there is a chemical variability between genotypes analyzed. The cassava genotypes showed substantial amounts of carotenoids, indicating their potential as source of interesting compounds to human health and nutrition, given the presence of pro-vitamin A carotenoids ( $\beta$-carotene, e.g.) and lycopene in roots of yellow and red color, respectively. The Rosada genotype was found to be discrepant because 


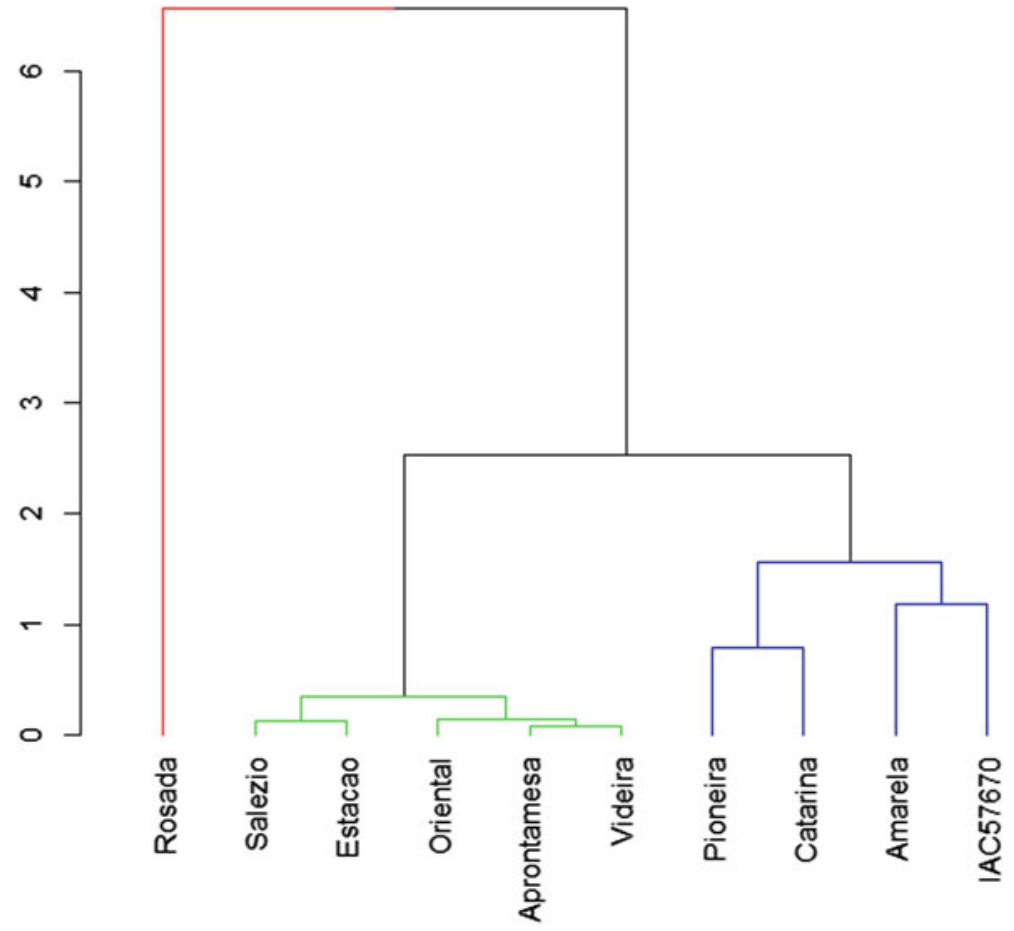

Fig. 5 Similarity of cassava genotypes in respect to their carotenoid composition determined by RP-HPLC, followed by hierarchical clustering analysis (UPGMA method - $97.61 \%$ of cophenetic correlation). The genotypes similarity between members of the same cluster is statistically significant $(\mathrm{p}<0.05)$ when the branches in the dendrogram show the same color. ${ }^{1}$

its richness in the carotenoids, in addition to the presence of lycopene in relevant amounts.

The information obtained by coupling the analysis of biochemical markers for provitamin A in cassava genotypes to bioinformatics tools revealed to be relevant for the rational design of biochemically-assisted cassava breeding programs. Indeed, the analytical approach adopted (i.e., UV-Vis/RP-HPLC/chemometrics) allowed to discriminate and classify the claimed genetic variability of the studied samples based on their biochemical traits, helping to identify/select cassava genotypes of interest to human health and nutrition.

Acknowledgements To FAPESC (Fundação de Amparo à Pesquisa e Inovação do Estado de Santa Catarina) and CNPq (Conselho Nacional de Desenvolvimento Científico e Tecnológico) for financial support. The research fellowship from $\mathrm{CNPq}$ on behalf of the later author is acknowledged.

\footnotetext{
${ }^{1}$ Significance determined by Simprof analysis (Similarity Profile Analysis) from R Clustsig package in accordance with Clarke, Somerfield \& Gorley (2008) [11].
} 


\section{References}

1. FAO (Food and Agriculture Organization of the United Nations): The global cassava development strategy and implementation plan, vol. 1, p. 70. Rome, Italy, 26-28 April 2000. http://www.fao.org/ag/agp/agpc/gcds/ (2012). Accessed 13 Apr 2012

2. WHO (World Health Organization): Global prevalence of vitamin A deficiency in populations at risk 1995-2005. WHO Global Database on Vitamin A deficiency, Geneva (2012)

3. Rodrigues-Amaya, D.B., Kimura, M., Amaya-Farfan, J.: Fontes brasileiras de carotenoides: tabela brasileira de composição de carotenoides em alimentos, p. 100. MMA (Ministério do Meio Ambiente), Brasília (2008)

4. Iglesias, C., Mayer, J., Chavez, L., Calle, F.: Genetic potential and stability of carotene content in cassava roots. Euphytica 94, 367-373 (1997)

5. Chavéz, A.L., Sánchez, T., Jaramillo, G., Bedoya, J.M., Echeverry, J., Bolaños, E.A., Ceballos, H., Iglesias, A.: Variation of quality traits in cassava roots evaluated in landraces and improved clones. Euphytica 143, 125-133 (2005)

6. Stahl, W., Sies, H.: Antioxidant activity of carotenoids. Mol. Aspects Med. 24, 345-351 (2003)

7. Rodriguez-Amaya, D.B., Kimura, M.: HarvestPlus handbook for carotenoid analysis. HarvestPlus Technical Monograph 2. International Food Policy Research Institute (IFPRI) and International Center for Tropical Agriculture (CIAT), Washington, DC, and Cali (2004)

8. R Core Team: R: a language and environment for statistical computing. R Foundation for Statistical Computing, Vienna, Austria. http://www.R-project.org/ (2015). ISBN 3-900051-07-0

9. Hanson, A.B.: ChemoSpec: an R package for chemometric analysis of spectroscopic data and chromatograms (Package Version 1.51-0) (2012)

10. Beleites, C.: Import and export of spectra files. Vignette for the R package hyperSpec (2011)

11. Clarke, K.R., Somerfield, P.J., Gorley, R.N.: Testing of null hypotheses in exploratory community analyses similarity profiles and biota-environment linkage. J. Exp. Mar. Biol. Ecol. 366, 56-69 (2008) 


\section{黛 Springer}

http://www.springer.com/978-3-319-19775-3

9th International Conference on Practical Applications of Computational Biology and Bioinformatics

Overbeek, R.; Rocha, M.P.; Fdez-Riverola, F.; De Paz, J.F.

(Eds.)

2015, XIII, 148 p. 53 illus., Softcover

ISBN: $978-3-319-19775-3$ 